\title{
Blocking as a function of added cue intensity
}

\author{
JEROME M. FELDMAN \\ Drake University, Des Moines, Iowa 50311
}

\begin{abstract}
Twelve rats were initially trained to leverpress for water in the presence of a tone. Responses in the absence of the tone were never reinforced. Following this pretraining, a novel light cue was simultaneously compounded with all presentations of the tone as a relevant but redundant predictor of reinforcement. For three different groups of subjects, the light cue was of either bright, moderate, or dim intensity. After compound training, all subjects experienced nonreinforced presentations of each of the two components of the compound. Results indicated that the relative degree of control exerted by the light over behavior increased with increases in light (added cue) intensity,
\end{abstract}

A number of investigators have been able to manipulate the relative degree of control over behavior exerted by cues contained in a simultaneous stimulus compound by modifying the amount of experience an organism has had with each of these cues prior to compound training (Feldman, 1971; Kamin, 1968, 1969). For example, Feldman (1971) first trained rats to respond for food in the presence of one cue (say, a tone) but not in its absence. Later, a novel cue (say, a light) was simultaneously compounded with all presentations of the original pretraining cue, thus forming a relevant-redundant stimulus compound, and compound-present/compound-absent discrimination training continued. Eventually, as a test for behavioral control, the cues in the compound were presented individually in extinction. Results indicated that not only did the added cue control behavior to a lesser extent than the pretraining cue, but that control by the added cue was less than if there had not been relevant pretraining prior to compound training. Kamin (1968) has labeled this procedure and effect "blocking" because the presence of the pretraining cue appears to block the acquisition of behavioral control ordinarily attainable by the added cue.

Although blocking may sometimes appear to be an all-or-none phenomenon, the degree of blocking may be influenced by a number of variables (see Feldman, 1971). For example, Kamin (1968), using rats in a conditioned suppression study, found that the size of the blocking effect decreased with decreases in the intensity of the auditory cue used as the discriminative stimulus during pretraining. Similar effects of pretraining cue intensity or difficulty have also been reported by others (Johnson \& Cumming, 1968; Mackintosh, 1965a). However, the results of these studies are difficult to

This research was partially supported by a grant from the Drake University Research Council given to the author. Requests for reprints should be sent to Jerome M. Feldman, Department of Behavioral Sciences, College of Optometry, State University of New York, 131 East 23rd Street, 7 th floor, New York, New York 10010. interpret because variations in intensity of the pretraining cue may also affect performance measures such as number of errors during pretraining, terminal level of pretraining performance, or number of errors during compound training. Thus, one is not sure whether pretraining cue intensity, perse, or differences in performance are responsible for the observed relationship between pretraining cue intensity and degree of blocking (Kamin, 1968). Indeed, Chase (1966) has shown that an inverse relationship exists between the magnitude of the blocking effect and the number of errors during compound training. Many of those studies (e.g., Kamin, 1968) which have reported an attenuation of the blocking effect using a low-intensity pretraining cue have also reported low levels of asymptotic performance during pretraining, as well as increases in asymptotic performance once the added cue has been introduced during compound training.

Given these difficulties of interpretation of effects of pretraining cue intensity, it would be interesting to manipulate added cue intensity. Under these conditions, differences in pretraining performance would not be expected to influence added cue control because all subjects would be exposed to the identical pretraining problem. However, it should be noted that even variations in added cue intensity could influence the magnitude of the blocking effect by directly affecting compound training performance through generalization decrement or external inhibition (Kamin, 1968). Another reason for manipulating added cue intensity was to assess whether manipulations other than those related to the pretraining cue could affect added cue control over behavior. This possibility has not been stressed by either of the two conceptualizations most capable of handling the blocking phenomenon-selective attention theory (Mackintosh, 1965b) or modified continuity theory (Rescorla \& Wagner, 1972). Each of these positions suggest that the primary determinant of added cue control is the amount of control acquired by pretraining cues prior to compound training. The role of the added cue is left relatively unspecified. 


\section{METHOD}

\section{Subjects}

Twenty-four male Long-Evans black-hooded rats served as subjects in the experiment. All subjects were experimentally naive and 75 days of age at the commencement of training. All subjects were housed in individual living cages and fed Purina Lab Blox ad lib each day. Immediately following each training session, the subjects were given a sufficient amount of tap water to maintain $80 \%$ of initial body weights (approximately $250 \mathrm{~g}$ ), corrected for normal growth-rate increases.

\section{Apparatus}

Training and testing were conducted in two noncommercial sound-attenuated operant chambers. Each chamber contained a houselight, cue light, liquid dipper system, manipulandum, and an 8-ohm speaker. A BRS audio oscillator (AO 201) and audio amplifier (AA 202) were used to produce a $3,000-\mathrm{Hz}$ tone at $85 \mathrm{~dB}$ through the speaker. Blowers ventilated the chambers and produced a masking noise in each chamber.

A $120-\mathrm{V} 7 \mathrm{~W}$ amber houselight was located $7.5 \mathrm{~cm}$ above the center of the top of each operant chamber. A white cue light was located $20.3 \mathrm{~cm}$ above the grid floor of each chamber, and was centered on the front panel of the chamber. The cue light $(3.8 \mathrm{~cm}$ in diam) was located directly above a $.05-\mathrm{cc}$ water dipper cup mechanism, the latter being $5.1 \mathrm{~cm}$ above the grid floor. The center of the response manipulandum was located $10.2 \mathrm{~cm}$ above the grid floor and $6.3 \mathrm{~cm}$ from the side of the chamber on the front panel. The 14.V .24-A cue light (Archer No. 1891) was wired in parallel with either no resistor, a $10-\mathrm{ohm}$ resistor, or a $40-\mathrm{hm}$ resistor, and corresponded to bright, medium, or dim-cue-light conditions, respectively. BRS solid state and electro-mechanical relay equipment controlled sequencing and timing of stimuli, reinforcement presentations, and recording of responses.

\section{Procedure}

All subjects were handled for 7 days prior to the commencement of training. During this time, the subjects were given a reduced ration of water so as to maintain body weight at approximately $80 \%$ of normal. Following this, the subjects were randomly assigned to one of the two operant chambers and were shaped to leverpress for $.05 \mathrm{cc}$ of water dispensed from a liquid dipper cup. Shaping was completed, on the average, in 7 days. All subjects then experienced 5 days of training on a variable interval 30-sec (VI 30) schedule of reinforcement followed by 2 days on a VI 60 schedule. All VI training was done in the absence of any tones, cue lights, or houselight.

Prior to experimental training, half of the 24 subjects were randomly assigned to a pretraining group and half to a no-pretraining control group. A third of the subjects $(N=4)$ in each of these major groups were randomly assigned to a bright-cue-light group; a third to a moderate-cue-light group; and, a third to a dim-cue-light group.

All subjects in the pretraining group $(\mathrm{N}=12)$ were given 32 days of MULT VI 60-Ext training. The houselight was not on for the remainder of the study. Each day's session" consisted of $36 \mathrm{~min}$; half of that time in the presence of a $3,000-\mathrm{Hz}$ tone and half in its absence. Cue presentations were of either 1 or $2 \mathrm{~min}$ duration. Responses were reinforced with $4 \mathrm{sec}$ access to the water dipper in the VI component only in the presence of the tone. Responses were never reinforced in the absence of the tone. No pretraining control subjects received no training at this time.

Following pretraining, all subjects received 32 days of compound training. Compound training followed the exact same pattern as pretraining, i.e., a MULT V1 60-Ext schedule of reinforcement, except that the cue in whose presence responses were reinforced consisted of a simultaneous relevant-redundant
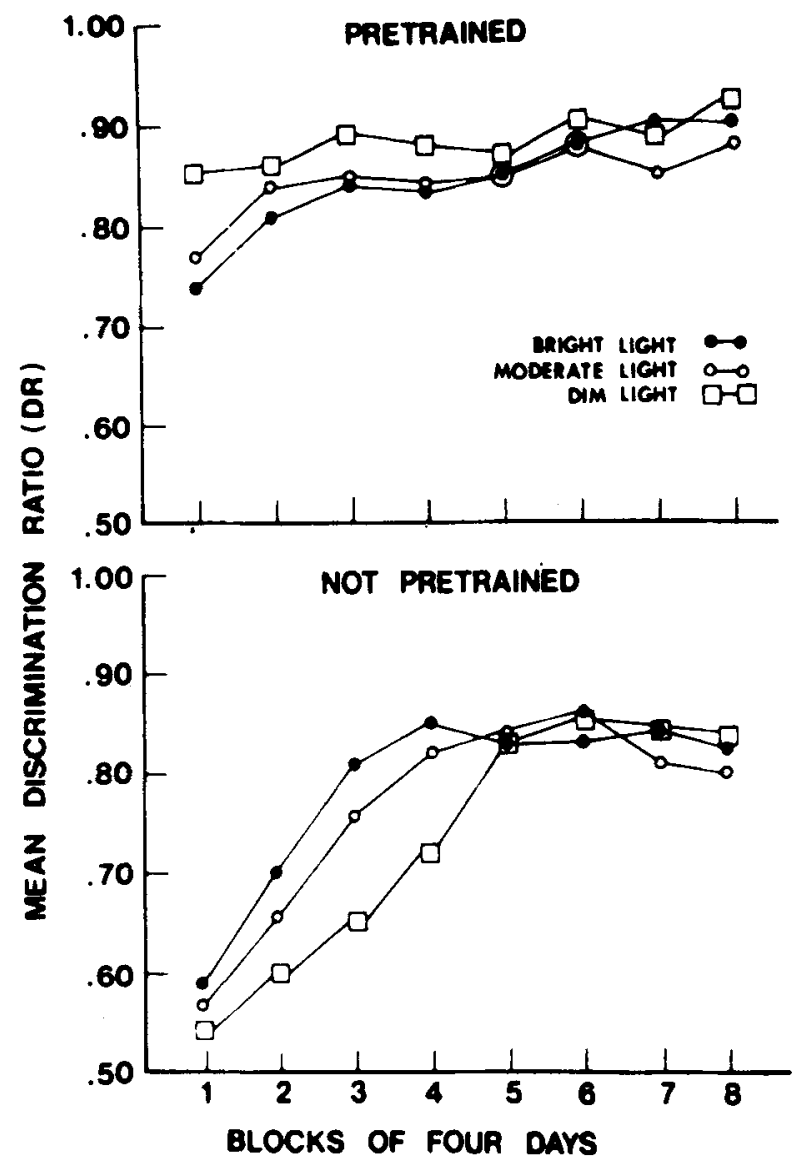

Figure 1. Mean discrimination ratio (DR) during compound training in blocks of 4 days, for groups exposed to bright, moderate, or dim added-cue light intensities. Upper and lower panels refer to performance of pretrained and nonpretrained groups, respectively.

compound of the original pretraining tone plus a visual cue light. As indicated previously, the intensity of the cue light varied for the three different pretraining subgroups and the three no-pretraining control groups. The cue light was of either bright, moderate, or dim intensity for each of the subgroups.

After compound training, all subjects experienced 2 days of testing in order to assess the degree of behavioral control accrued by each of the components of the reinforced compound, i.e., the tone cue and the light cue. Each day's test session began with a 6-min "warm-up" phase during which MULT Vl 60-Ext compound training continued. During the remainder of the test session, responses were never reinforced. During that time, the compound training cue light and tone were each presented individually in random order (in 1-or 2-min segments) for a total of 5 min of each. Interspersed between cue presentations (in 1 or 2-min segments) was a total of $10 \mathrm{~min}$ of the absence of the cues.

\section{RESULTS}

During both pretraining and compound training, the daily performance of each subject was assessed in terms of a discrimination ratio (DR); the number of responses made in the presence of the reinforced cue, $\mathrm{S}+$, divided by the total number of responses made in the presence of $\mathrm{S}+$ and the nonreinforced cue, $\mathrm{S}-$. 
An analysis of variance revealed that there was no significant difference between any of the pretraining groups during pretraining. However, DR performance did improve for all groups, across 4-day blocks of pretraining, from a value of .54 to a value of .77 , $F(7,63)=114.23, p<.001$. Upper and lower panels of Figure 1 depict the mean DR, in blocks of 4 days, for pretrained and nonpretrained groups during compound training, respectively. As can be seen, the performance of all nonpretrained groups improved across blocks of compound training days, $F(7,63)=79.78, p<.001$, with all groups achieving approximately the same asymptotic level of performance, a DR of .80 . However, the rate of this improvement was faster for groups experiencing a compound containing the bright-light cue rather than the dim-light cue, $F(14,63)=3.61, p<.005$. Pretrained groups also showed significant overall improvement in performance during compound training, $F(7,63)=21.43, p<.001$, with terminal levels of performance being better than terminal pretraining performance, $t, d f=11, p<.001$. While all pretrained groups showed about the same asymptotic DR (.90) during compound training, the rate at which this level was achieved differed between groups, $F(14,63)=2.14$, $p<.05$. Unlike the nonpretrained groups, where increases in cue light intensity facilitated performance, the performance of the pretrained bright-cue-light group was initially lower than that of the pretrained dim-cue-light group. In order to further analyze this finding, terminal pretraining performance was compared to initial compound training performance. This was done by computing the number of responses made by each

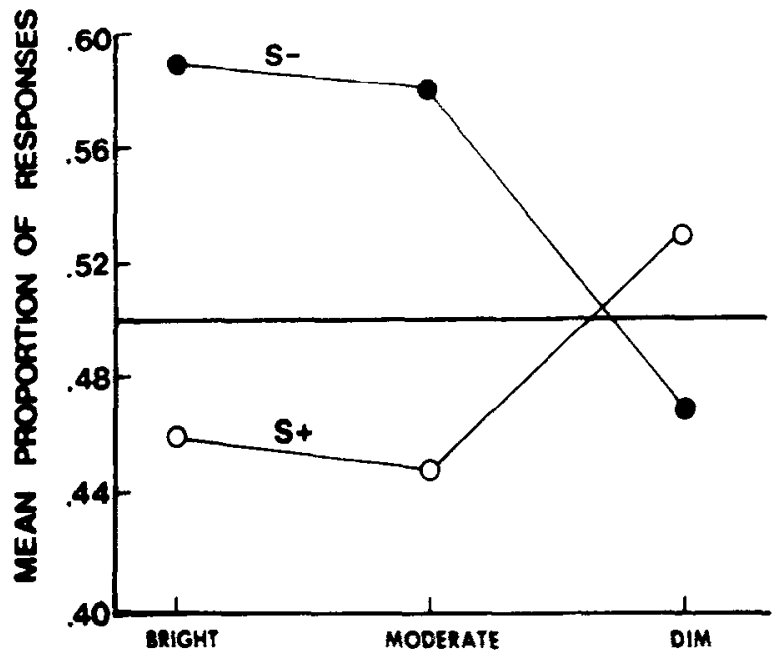

Figure 2. Mean proportion change in responding in the presence of $S+$ or $S-$ cues as a function of added cue light intensity for three pretrained groups. The performance measure was computed by taking the mean number of responses made in the presence of $S+$ (or $S_{-}$) on the last block of pretraining and dividing it by that same number plus the mean number of responses made in the presence of $S+$ (or $S-$ ) on the 1st day of compound training.

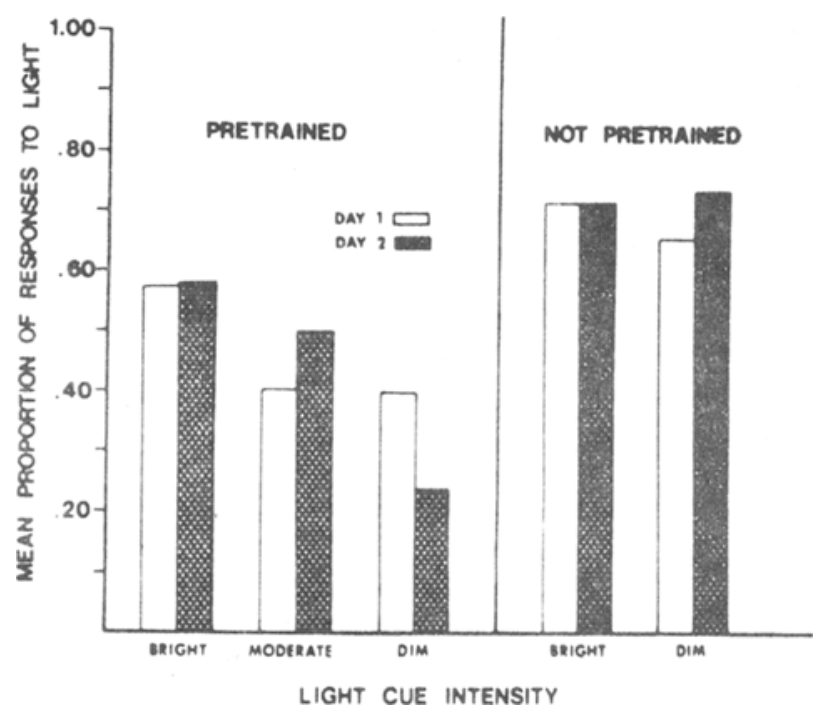

Figure 3. Mean percentage of responses made during testing to the light cue as a function of added light-cue intensity experienced during compound training (and testing) and test day. The performance measure was computed by taking the mean number of responses made in the presence of the light cue in 5 min of daily testing and dividing it by that same number plus the mean number of responses made in the presence of the tone cue in $5 \mathrm{~min}$ of daily testing.

subject in the presence of S+ during the last block of pretraining and comparing it to the number of responses made during the first day of compound training. A similar ratio was computed using the number of responses made during $\mathrm{S}-$ presentations. As can be seen from Figure 2, pretrained subjects, in general, made a smaller proportion of responses in the presence of $\mathrm{S}+$ during compound training compared to pretraining; and, a higher proportion of responses in the presence of $S^{-}$ during compound training compared to pretraining, $F(1,9)=17.77, p<.005$. However, this effect held only for pretrained subjects experiencing either the bright or moderate cue light during compound training, $F(2,9)=31.71, \quad p<.001$. The dim-cue-light group responded about the same to $S+$ and $S-$ during terminal pretraining and initial compound training.

Following compound training, a 2-day test was conducted for all subjects in which the individual cues comprising the compound were presented individually. Figure 3 depicts the mean percentage of responses made in the presence of the light, compared to the tone, for the three pretrained groups and two of the nonpretrained groups (due to an equipment failure, the test performance of the nonpretrained moderate-cuelight group was lost). As is apparent from Figure 3, both of the nonpretrained groups emitted about $70 \%$ of the total number of responses made during testing to the light cue, and there was no significant difference between the bright- and dim-light groups. However, the performance of the pretrained groups did differ as a 
function of the cue ligint intensity experienced during compound training. As can be seen from Figure 3, while all of the pretrained groups made less than $70 \%$ of their test responses to the light cue, the greater the intensity of the light cue experienced during compound training, the greater the percentage of responses made to the light cue during testing, $F(2,9)=6.87, p<.025$. In addition, this effect was even more pronounced on Test Day 2 than on Test Day 1 for bright- and dim-cue-light groups, $F(2,9)=6.09, p<.025$.

\section{DISCUSSION}

The major finding in the present study was that added cue intensity appeared to affect the degree of control over behavior later exhibited by the added cue. That is, subjects which experienced an intense added cue during compound training and testing distributed a greater proportion of the total responses made during testing to that cue than did subjects which experienced a less intense added cue during compound training and testing. In addition, when compared to the performance of nonpretrained subjects, the blocking effect decreased as a function of increases in added cue intensity for pretrained subjects. These findings seem to be inconsistent with the general assumptions used to account for blocking results by both modified continuity theory (Rescorla \& Wagner, 1972) and selective attention theory (Mackintosh, 1965b).

Both modified continuity theory and selective attention theory imply that any behavioral control which might be accrued by the added cue of a stimulus compound in the blocking paradigm should be determined by the amount of behavioral control already accrued by the pretraining cue prior to compounding. The more behavioral control accrued by the pretraining cue, the less left to be accrued by the added cue. The only specified function of cue salience is in modified continuity theory. The assumption there is that cue salience partially determines (along with the magnitude of the reinforcing event) the rate at which this behavioral control will be acquired. However, once the asymptotic amount of behavioral control has been acquired, the role of cue salience diminishes. Thus, if it is assumed in the present experiment that the pretraining cue has maximal behavioral control, then variations in added cue intensity should exert little influence upon added cue control.

While the present data seem to implicate added cue intensity as a factor affecting eventual added cue control over behavior in the blocking paradigm, the source of this effect is more difficult to identify. For example, while the terminal pretraining performance of all subjects was about the same (as predicted), terminal compound training performance was significantly higher, with subjects trained with the less intense added cue reaching that level sooner than subjects trained with the more intense added cue. The fact that the difference in performance between groups trained with different-intensity added cues occurred primarily at the onset of cornpound training (see Kamin, 1968) in no way diminishes the fact that groups experiencing the more intense added cue made more errors during compound training than groups experiencing the less intense added cue. As Chase (1966) has suggested, the magnitude of the blocking effect may decrease with increases in the number of errors made during compound training. Thus, number of errors made during compound training, rather than any inherent effect of added cue intensity (possibly affecting the discriminability of periods of reinforcement from nonreinforcement during compound training), may have been responsible for the observed test performance. Moreover, the test results may have been partially influenced by the particular added-cue intensity experienced during testing, with increases in intensity yielding increased probabilities of responding. Although this possibility is unlikely because it did not differentially affect the added-cue test performance of nonpretrained groups, it cannot entirely be ruled out as a selective factor.

Perhaps, an appropriate test to dissociate the confounding between compound-training error rates and added-cue discriminability would be to introduce the added cue during pretraining as an irrelevant cue, making it relevant later at the onset of compound training. By this method, the disruption in performance at the onset of compound training in the present experiment, presumably due to the introduction of a novel cue, might be eliminated. Of course, differential error rates during pretraining and compound training, reflecting differences in added-cue intensity, would also have to be considered. Nevertheless, if similar blocking results were observed, then one might be more able to strongly assert the importance of the discriminability function of added cue intensity, rather than behavioral disruption, as a primary factor in blocking.

Despite the just-mentioned considerations, the data do indicate that as added cue intensity increases, the blocking effect (evaluated by comparing the test performance of pretrained and nonpretrained groups) decreases. Moreover, the proportion of behavioral control distributed to the pretraining and added cues of the pretrained groups also changes. The model proposed by Rescorla and Wagner (1972) might possibly be extended to handle the present findings in the following manner: If the behavioral disruption which occurred at the onset of compound training for the pretrained group experiencing the most intense added cue was taken to mean that the excitatory value of the stimulus compound was momentarily depressed below asymptote, then the added cue would be expected to gain excitatory strength when reinforcement occurred. It could be assumed that this gain would be proportional 
to the discrepancy between current excitatory value and asymptote. Thus, the more disruption, the more gain. This would account for the attenuated blocking effect observed for the pretrained group experiencing the most intense added cue, i.e., the cue which yielded the greatest amount of behavioral disruption. In addition, since the rate of increment in excitatory strength is assumed to increase with increases in cue salience, then it might also be expected that the rate of gain of excitatory strength for pretraining and added cues would be unequal and, therefore, disproportionate. Thus, the relative degree of behavioral control accrued by the added cue might even be more than the pretraining cue, as was observed in the present experiment.

\section{REFERENCES}

Chase, S. The effects of discrimination training on the development of stimulus control by single dimensions of a stimulus compound. (Doctoral dissertation, City University of
New York) Ann Arbor, Mich: University Microfilms, 1966, No. 66-11-385.

Feldman, J, M. Added cue control as a function of reinforcement predictability. Journal of Experimental

Psychology, 1971, 91, 318-325.
Johnson, D. F., \& Cumming, w. W. Some determiners of attention. Journal of the Experimental Analysis of Behavior, $1968,11,157-166$.

Kamin, L. J. Attention-like processes in classical conditioning. In M. R. Jones (Ed.), Miami symposium on the prediction of behavior 19677: Aversive stimulation. Coral Gables: University of Miami Press, 1968.

Kamin, L. J. Selective association and conditioning. In W. K. Honig and N. J. Mackintosh (Eds.), Fundamental issues in associative learning. Halifax: Dalhousie University Press, 1969.

Mackintosh, N. J. Incidental cue learning in rats. Quarterly Journal of Experimental Psychology, 1965a, 17, 292-300.

Mackintosh, N. J. Selective attention in animal discrimination learning. Psychological Bulletin, 1965b, 64, 124-150.

Rescorla, R. A., \& Wagner, A. R. A theory of Pavlovian conditioning: Variations in the effectiveness of reinforcement and nonreinforcement. In A. H. Black and W. F. Prokasy (Eds.), Classical conditioning II: Current theory and research. New York: A ppleton-Century-Crofts, 1972.

(Received for publication July 11, 1974: revision received September 30, 1974.) 